\title{
Application of Nucleic Acid Reference Material for Rapid Detection of Cronobacter sakazakii (Cronobacter spp.) in Flammulina velutipes
}

\author{
Huandie Yang $(\mathbb{D}){ }^{1,2}$ Danyang Guo $\left(\mathbb{D},{ }^{1}\right.$ Leilei Chen $(\mathbb{D}),{ }^{1}$ Alexander Suvorov ${ }^{(D)}$,

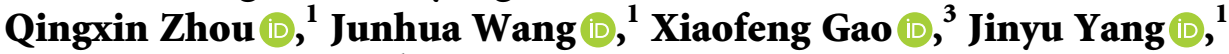 \\ and Xiangyan Chen (iD) \\ ${ }^{1}$ Institute of Agro-Food Science and Technology, Shandong Academy of Agricultural Sciences, \\ Key Laboratory of Agro-Products Processing Technology of Shandong Province, \\ Key Laboratory of Novel Food Resources Processing, Ministry of Agriculture and Rural Affairs, Jinan 250100, China \\ ${ }^{2}$ College of Life Science, Shandong Normal University, Jinan 250014, China \\ ${ }^{3}$ National $R$ \& D Center for Edible Fungus Processing Technology, Henan University, Kaifeng 475004, China
}

Correspondence should be addressed to Jinyu Yang; yangjinyu86@163.com and Xiangyan Chen; 315478845@qq.com

Received 30 July 2020; Revised 9 October 2020; Accepted 26 October 2020; Published 18 November 2020

Academic Editor: Fatma M. El-Demerdash

Copyright $(92020$ Huandie Yang et al. This is an open access article distributed under the Creative Commons Attribution License, which permits unrestricted use, distribution, and reproduction in any medium, provided the original work is properly cited.

Cronobacter sakazakii (C. sakazakii) widely exists in the environment and is a common foodborne pathogenic microorganism. It can cause meningitis, bacteremia, and necrotizing enterocolitis in infants and elderly people with low immunity. Therefore, the development of the rapid detection method for foodborne pathogen C. sakazakii is very important for food safety control. This study has developed a kind of genomic nucleic acid reference material as a positive reference for the rapid detection of $C$. sakazakii in the edible fungus Flammulina velutipes. First, the genomic nucleic acids were extracted from the standard strain of $C$. sakazakii, and the nucleic acids were lyophilized. The uniformity test result of the lyophilized powder showed that there were no significant differences between samples. In short-term stability tests at $4^{\circ} \mathrm{C}$ and $37^{\circ} \mathrm{C}$ for 14 days, the properties of the nucleic acid reference material were good, and in long-term stability tests at $4^{\circ} \mathrm{C}$ and $-20^{\circ} \mathrm{C}$ for 8 months, the samples are still stable. To verify the application of this nucleic acid reference material, it was used as a positive control template for PCR detection and applied to the rapid detection of $C$. sakazakii in $F$. velutipes. It was found that 13 of the 15 F. velutipes samples were positive for C. sakazakii. As far as we know, this is the first attempt to detect C. sakazakii in F. velutipes using the PCR method with nucleic acid reference material as the positive reference. This study is helpful in promoting the extensive and in-depth application of nucleic acid reference materials in foodborne pathogens detection.

\section{Introduction}

Foodborne pathogens are pathogenic bacteria that can cause food poisoning or use food as a medium [1]. They threaten human health and can cause serious food safety problems [2]. There are many types of food pathogens, such as diarrheagenic Escherichia coli, Staphylococcus aureus, Salmonella spp., Listeria monocytogenes, and Enterobacter sakazakii. Cronobacter sakazakii, previously called Enterobacter sakazakii, is an facultative anaerobic Gram-negative
Bacillus. This pathogenic bacterium could be isolated from a wide variety of foods and has been detected in infant milk powder, meat, water, vegetables, and other foods $[3,4]$. Infants and young children are high-risk groups of Cronobacter infections $[3,5]$. The infections mainly cause bacteremia, meningitis, and necrotizing enterocolitis, and the fatality rate is as high as $50 \%-80 \%$ [6]. Edible fungi may be contaminated by foodborne pathogens during planting, harvesting, packaging, and transportation. It is reported that pathogenic bacteria, such as L. monocytogenes, have been 
detected in edible fungi $[7,8]$. Cronobacter spp. also could be detected in dried edible macrofungi samples [9]. A recent study showed that the detection rate of Cronobacter spp. is $17.54 \%$ (37 positive in 211 samples) in Flammulina velutipes from 44 cities in China [10], indicating that the detection of $F$. velutipes contamination should be strengthened to avoid food safety issues.

The traditional detection method of food pathogenic microorganisms is to conduct biochemical identification after enrichment and cultivation of bacteria in the tested sample [11]. However, this detection method has cumbersome steps and a long time-consuming process, which cannot meet the requirements of rapid foodborne pathogen detection. Therefore, the establishment of accurate, sensitive, rapid, and specific detection methods for the detection of food pathogenic microorganisms could play an important role in the prevention and control of food poisoning events. At present, a variety of rapid detection methods have been developed, these methods are mainly polymerase chain reaction (PCR) method and loop-mediated isothermal amplification (LAMP) method based on molecular biology [12], and enzyme-linked immunosorbent assay (ELISA) based on immunology [13]. Among them, the most commonly used methods based on molecular biology are ordinary PCR and fluorescent quantitative PCR $[1,14]$. And ordinary PCR is widely used in food inspection department because of its easiness to operate with simple steps, short time-consuming, and easy to conduct [1]. The common point of these detection methods is that they require DNA reference materials as reaction templates. The template can be genomic DNA of pathogenic bacteria or plasmid carrying characteristic virulence genes. So the preparation of nucleic acid reference materials of standard strains of foodborne pathogens as positive control substances could play an important role in rapid detection of foodborne pathogens.

In order to provide a positive reference material for the rapid detection of $C$. sakazakii in food inspection, the study of ready-to-use $C$. sakazakii nucleic acid reference materials was conducted in this paper, and its application in detection of $F$. velutipes was also performed. The research results not only provides a positive reference material but also greatly shortens the detection time, saves the cost, and improves the detection accuracy.

\section{Materials and Methods}

2.1. Strains and Culture Condition. Cronobacter sakazakii FC4146 (CMCC 45404) was obtained from National Institute for Food and Drug Control of China. For genome extraction, the bacterial strains were cultured in BHI, and for enrichment of C. sakazakii, the samples to be detected were cultured in modified lauryl sulfate tryptose broth-vancomycin medium (mLST-Vm).

\subsection{Preparation of the Genomic DNA Reference Materials of C. sakazakii}

2.2.1. Genomic DNA Extraction of C. sakazakii. The bacterial strain was cultured in BHI for $12-16 \mathrm{~h}$, and the bacteria were collected by centrifugation at $8,000 \mathrm{rpm}$ for $10 \mathrm{~min}$ at $4^{\circ} \mathrm{C}$. The genome extraction was conducted according to the manufacturer's protocol of EasyPure Bacteria DNA Kit (TransGEN, Beijing), and the genome samples were stored at $-20^{\circ} \mathrm{C}$.

2.2.2. Determination of Genomic DNA Concentration and Purity. One microliter of genomic DNA sample was applied to Thermo NanoDrop 2000/2000 c to measure the purity and concentration of genomic DNA.

\subsubsection{Detection of Characteristic Sequence of C. sakazakii.} The characteristic sequence of C. sakazakii (16S-23S) was determined according to determination of Enterobacter sakazakii (Cronobacter spp.) from dehydrated powdered milk for export. Part 2: the PCR method with some modifications (SN/T 1632.2-2013; the People's Republic of China Entry-Exit Inspection and Quarantine Industry Standard, 2013). The primers for amplification of 16S-13S were forward primer, GGGTTGTCTGCGAAAGCGAA, and reversed primer, GTCTTCGTGCTGCGAGTTTG. The PCR reaction system (Table S1) and PCR condition (Table S2) are shown in the supplementary materials.

2.2.4. Detection of the Integrity and Purity of Genomic DNA of C. sakazakii. After PCR, $5 \mu \mathrm{L}$ of genomic DNA was loaded onto a $1 \%$ agarose gel (containing dye GoldView), and the electrophoresis was conducted at $175 \mathrm{~V}$ constant pressure for $25 \mathrm{~min}$, in the buffer of $1 \times \mathrm{TBE}$. The integrity and purity of genomic DNA was observed under UV detection in a gel imager.

2.3. PCR Specificity and Sensitivity Evaluation. For specificity evaluation, the genomic DNA of Escherichia coli O157 : H7 (CMCC 44939), E. coli (CICC 10003), Staphylococcus aureus (CMCC 26301), Salmonella enteritidis (CMCC 50957), Bacillus amyloliquefaciens (CCTCC M2013098), Bacillus subtilis (CGMCC No. 14221), Lactobacillus casei (CGMCC No. 14249), Enterococcus faecium (ATCC 29212), Alcaligenes faecalis (CCTCC M2017497), and Listeria monocytogenes (CMCC 54008) were extracted according to the protocol of EasyPure ${ }^{\circledast}$ Genomic DNA Kit (TransGEN, Beijing), and the genomic DNA were used as templates to amplify the 16S-23S with the same method above, and sterilized water was used as the negative control. The PCR products were analyzed by $1.0 \%$ of agarose gel electrophoresis.

For sensitivity evaluation, the genomic DNA of C. sakazakii were diluted with sterilized water with gradients of $10^{-1}, 10^{-2}, 10^{-3}, 10^{-4}, 10^{-5}$, and $10^{-6}$. And the diluted samples were applied as templates to amplify $16 \mathrm{~S}-23 \mathrm{~S}$. The negative control used sterilized water as template in the reaction. The assessment of PCR products was conducted by $1.0 \%$ of agarose gel electrophoresis. 
2.4. Preparation of Lyophilized Powder of Genomic DNA Reference Material of C. sakazakii. The genomic samples of C. sakazakii were extracted and diluted to $20 \mathrm{ng} / \mu \mathrm{L}$; then, the samples were sent to Chinese Academy of Inspection and Quarantine for lyophilization, with $300 \mu \mathrm{L}$ of genomic DNA each ampoule. The lyophilized powder was stored at $-20^{\circ} \mathrm{C}$.

\subsection{Homogeneity Determination of Genomic DNA Reference} Material of C. sakazakii. Among the 200 bottles of genomic DNA reference materials, 12 bottles were randomly selected, and $300 \mu \mathrm{L}$ of sterile $\mathrm{ddH}_{2} \mathrm{O}$ was added to hydrate the lyophilized powder; then, $1 \mu \mathrm{L}$ sample was used to determine the concentration, and each sample was tested under the same condition twice. The test data were processed by SPSS with single factor statistical analysis. And the characteristic sequence $16 \mathrm{~S}-23 \mathrm{~S}$ was determined for each sample.

\subsection{Short-Term Stability Test of Genomic DNA Reference} Material of $C$. sakazakii. The short-term storage stability tests were conducted under the condition of $4^{\circ} \mathrm{C}$ and $37^{\circ} \mathrm{C}$. Forty-two bottles are randomly selected and tested three samples on $1,3,5,7,9,11$, and 14 days at $4^{\circ} \mathrm{C}$ and $37^{\circ} \mathrm{C}$, respectively. The concentration and the characteristic sequence $16 \mathrm{~S}-23 \mathrm{~S}$ were determined.

2.7. Long-Term Stability Test of Genomic DNA Reference Material of C. sakazakii. Thirty bottles of genomic DNA samples were selected randomly from the lyophilized powder samples, and 15 samples were stored at $4^{\circ} \mathrm{C}$, and the other samples were stored at $-20^{\circ} \mathrm{C}$. The concentration and characteristic 16S-23S sequence amplification tests were conducted at 7 time points in the first, second, fourth, sixth, eighth, and tenth months, respectively.

\subsection{Application of Genomic DNA Reference Material of C. sakazakii in Edible Fungus Flammulina velutipes}

2.8.1. Sampling of Edible Fungus Flammulina velutipes. Thirty samples of fresh Flammulina velutipes were collected from supermarkets and traditional retail markets in July 2020 from Jinan, China. While the samples were obtained, the analyses were managed immediately.

2.8.2. Determination of C. sakazakii in the Edible Fungus Flammulina velutipes. The needle mushroom (Flammulina velutipes) was obtained from the market and put into the sterile sealed bag in the low temperature insulation box. As the sample arrived at the laboratory, the detection was started to avoid additional pollution. The needle mushroom was taken out from the bag in the aseptic operation table, cut into pieces, and placed into the mLST-Vm enrichment medium. The enrichment of $C$. sakazakii was conducted according to the National Food Safety Standard of China Food microbiological examination: Enterobacter sakazakii for powdered infant formula [15]. After enrichment of bacteria, the culture was treated by boiling waterbath for $10 \mathrm{~min}$ to release the genomic DNA. Then, the genomic
DNA was taken as the template to amplify the characteristic $16 S-23 S$ sequence. The PCR products were analyzed by $1 \%$ agarose gel electrophoresis.

2.8.3. Verification of the Detection of C. sakazakii in the Edible Fungus Flammulina velutipes. The Flammulina velutipes samples that were positive for the $16 \mathrm{~S}-23 \mathrm{~S}$ sequence of $C$. sakazakii detected by PCR were identified according to the method of the National Food Safety Standard of China Food microbiological examination: Enterobacter sakazakii for powdered infant formula [15]. First, 1 loop of the enriched culture was taken and streaked into two chromogenic C. sakazakii agar plates and cultivated at $37^{\circ} \mathrm{C}$ for $24 \mathrm{~h}$. Then, at least 5 suspicious colonies were chosen; if there were less than 5 , all the suspicious colonies were taken, inoculated them on the TSA plate, and incubated at $25^{\circ} \mathrm{C}$ for $48 \mathrm{~h}$. Then, the yellow suspicious colonies on the TSA plates were conducted by biochemical identification in the $C$. sakazakii identification reagent strip.

\section{Results}

3.1. Preparation of Genomic DNA of C. sakazakii. The genomic DNA of C. sakazakii was extracted following the steps in the kit instructions, and the concentration and purity of DNA samples were determined using an ultraviolet spectrophotometer (Table 1), and the integrity was analyzed by agarose gel electrophoresis (Figure 1). The concentration of the genomic DNA of C. sakazakii was approximately $50 \mathrm{ng} /$ $\mu \mathrm{L}$, and the purity index A260/A280 was more than 1.7, indicating that the sample was not contaminated by protein. Also on the agarose gel, it could be observed that the genomic DNA band is single and bright, no protein and RNA contamination, consistent with the purity test results, showing that the purity and concentration of the extracted DNA are good, meeting the requirements of subsequent experiments.

3.2. Specificity Analysis of Primers of $16 S-23 S$. The specificity verification of the primers of the characteristic sequence 16S-23S was performed, and the result is shown in Figure 2. There was a bright band ( $282 \mathrm{bp}$ ) on the gel while using the genomic DNA of C. sakazakii FC4146 as the template and applying primers of $16 \mathrm{~S}-23 \mathrm{~S}$, but no bands could be observed when using other bacterial genomic DNA detected, indicating that the primers for amplifying the sequence of 16S-23S of C. sakazakii is specific.

3.3. Sensitive Analysis of Primers of 16S-23S Amplification of C. sakazakii. The sensitive analysis of the primers for characteristic sequence $16 \mathrm{~S}-23 \mathrm{~S}$ was detected. The genomic DNA was diluted by gradient dilution, and PCR reaction was conducted using the diluted DNA as template and applying the primers of $16 \mathrm{~S}-23 \mathrm{~S}$, and each gradient concentration was detected twice. As shown in Figure 3, the bands of PCR products were observed when the template content was greater than or equal to $0.004 \mathrm{ng}$, illustrating that the 
TABLE 1: The concentration and purity of genomic DNA of C. sakazakii.

\begin{tabular}{lcccc}
\hline Tube no. & 1 & 2 & 3 & 4 \\
\hline C $(\mathrm{ng} / \mu \mathrm{L})$ & 51.0 & 49.7 & 51.2 & 52.6 \\
$\mathrm{~A} 260 / \mathrm{A} 280$ & 1.78 & 1.77 & 1.78 & 1.80 \\
\hline
\end{tabular}

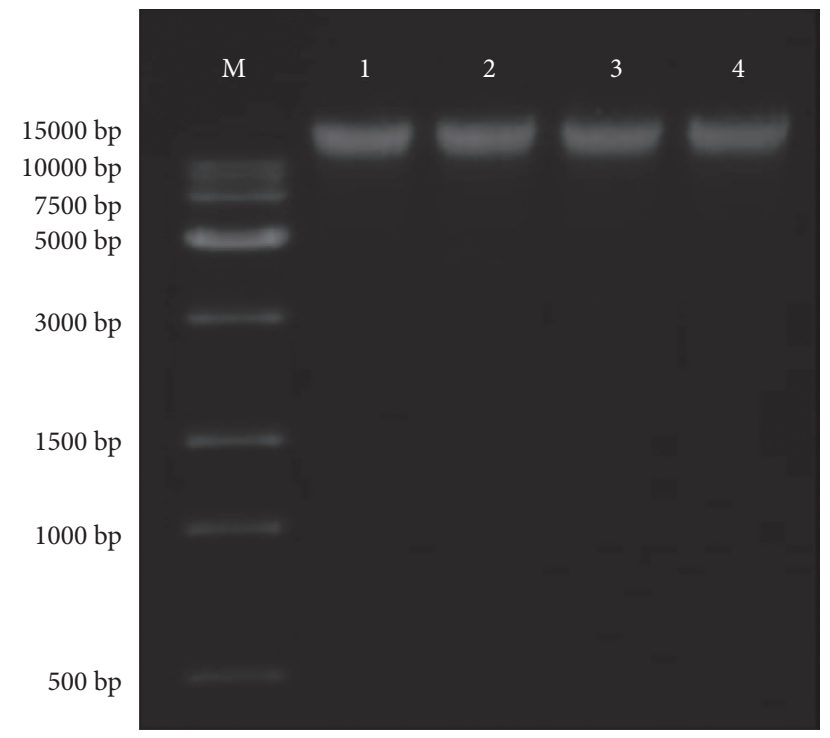

FIGURE 1: Agarose gel electrophoresis analysis of the genomic DNA of C. sakazakii. $M$, trans15K DNA marker; lanes 1-4, genomic DNA of C. sakazakii.

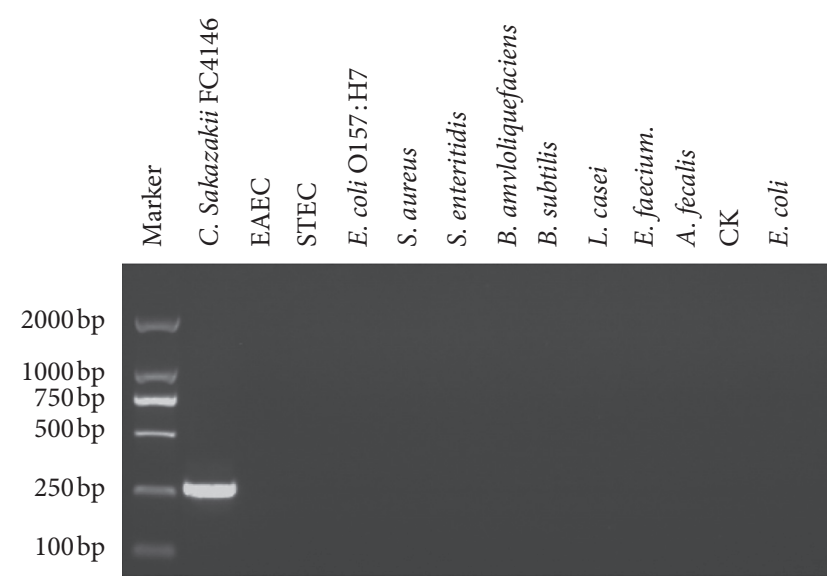

Figure 2: Specificity analysis of $16 \mathrm{~S}-23 \mathrm{~S}$ PCR result.

detection sensitivity of primers of $16 \mathrm{~S}-23 \mathrm{~S}$ was as low as $0.004 \mathrm{ng}$, also showing that the detection limit of the primers applied was at the $4 \mathrm{pg}$ level using genomic DNA of C. sakazakii as the template.

3.4. Uniformity Analysis of Genomic DNA Reference Materials of C. sakazakii. After the genomic samples were extracted, the samples were divided into $300 \mu \mathrm{L}$ each bottle, $6 \mu \mathrm{g}$ in total for lyophilization, and the total number of samples was 200 bottles. Each bottle is an indivisible whole unit, so 15 samples were randomly selected for uniformity

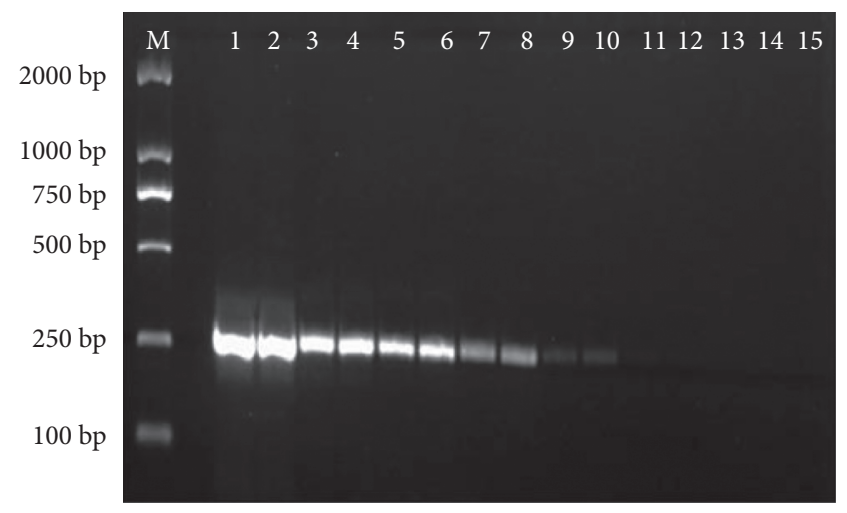

Figure 3: Sensitivity analysis of primers for $16 \mathrm{~S}-23 \mathrm{~S}$. $M$, DNA marker D (100-2000 bp); lanes 1-2, $40 \mathrm{ng}\left(10^{0}\right)$; lanes 3-4, $4 \mathrm{ng}$ $\left(10^{-1}\right)$; lanes 5-6, $0.4 \mathrm{ng}\left(10^{-2}\right)$; lanes $7-8,0.04 \mathrm{ng}\left(10^{-3}\right)$; lanes 9-10, $0.004 \mathrm{ng}\left(10^{-4}\right)$; lanes $11-12,0.0004 \mathrm{ng}\left(10^{-5}\right)$; lanes 13-14, $0.00004 \mathrm{ng}\left(10^{-6}\right)$; and lane 15 , blank control.

TABLE 2: Statistical analysis of homogeneity detection of genomic DNA reference material of C. sakazakii.

\begin{tabular}{lccc}
\hline Tube no. & Content 1 $(\mu \mathrm{g})$ & Content 2 $(\mu \mathrm{g})$ & Content 3 $(\mu \mathrm{g})$ \\
\hline 1 & 6.06 & 5.94 & 6.18 \\
2 & 5.85 & 6.03 & 5.94 \\
3 & 5.97 & 5.85 & 6.06 \\
4 & 6.06 & 5.94 & 5.85 \\
5 & 6.06 & 5.91 & 6.03 \\
6 & 5.91 & 6.00 & 6.06 \\
7 & 6.00 & 5.94 & 6.06 \\
8 & 6.12 & 5.97 & 5.94 \\
9 & 6.06 & 5.88 & 6.09 \\
10 & 6.06 & 6.12 & 5.97 \\
11 & 5.91 & 6.09 & 6.09 \\
12 & 6.00 & 5.91 & 6.06 \\
13 & 5.79 & 6.09 & 6.09 \\
14 & 5.88 & 5.85 & 6.24 \\
15 & 6.09 & 5.91 & 6.15 \\
Mean $(\mu \mathrm{g})$ & \multicolumn{3}{|c}{$6.00 \pm 0.1$} \\
$F$ value & \multicolumn{3}{c}{0.289} \\
$F$ critical value & \multicolumn{3}{c}{$(14,30)=2.04$} \\
\hline
\end{tabular}

inspection. The contents were detected using a UV spectrophotometer, and the statistical analysis of the detection result was conducted by the variance analysis method of one-way ANOVA. Three individual tests were performed for each sample. As shown in Table 2, the average content for 15 samples was $6.00 \mu \mathrm{g}$, and the variance of the determination results showed that with $95 \%$ confidence probability, $F$ value, 0.289 , was less than the $F$ critical value, 2.04, indicating that there is no significant difference between samples. And the characteristic sequence $16 \mathrm{~S}-23 \mathrm{~S}$ amplification test showed that the PCR products were positive and had a single band on the agarose gel for each test (Figures 4 and 5). Both the results indicate that the uniformity of the sample meets the requirements of the reference material.

3.5. Short-Term Stability Analysis of Genomic DNA Reference Material of C. sakazakii. The genomic DNA reference 


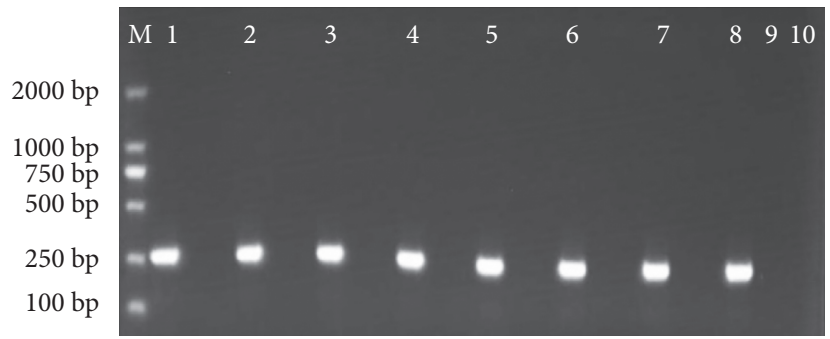

Figure 4: The PCR test for genomic DNA reference materials of no. 1-8. M, DNA marker D (100 2000 bp); lanes 1-8, PCR results of no. $1-8$, respectively; lane 9 , negative control; and lane 10 , blank control.

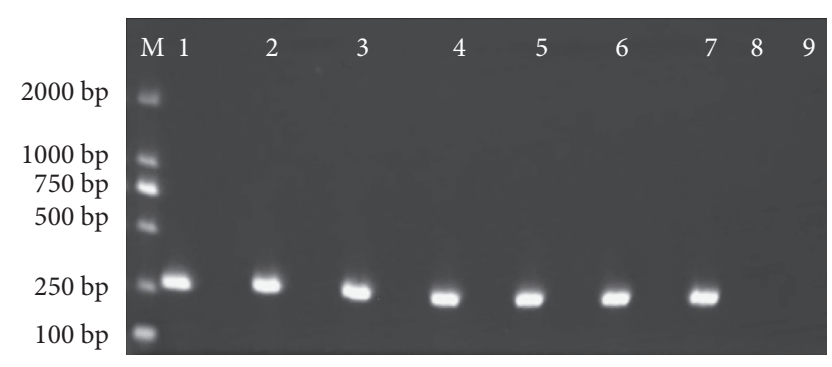

FIGURE 5: The PCR test for genomic DNA reference materials of no. 9-15. $M$, DNA marker D (100 $2000 \mathrm{bp})$; lanes 1-7, PCR results of no. 9-15, respectively; lane 8 , negative control; and lane 9, blank control.

materials were stored at $4^{\circ} \mathrm{C}$ and $37^{\circ} \mathrm{C}$ for a period of $1,3,5,7$, 9,11 , and 14 days, respectively; then, the concentration of each sample was detected, and the contents of genomic DNA were calculated, and statistical analysis by one-way ANOVA was conducted. The $F$ value (0.485) was less than the critical value (1.53) at $95 \%$ confidence, and the contents changes of nucleic acid reference material are shown in Figure 6, showing that the genomic nucleic acid reference material was stable for 14 days stored at $4^{\circ} \mathrm{C}$ or $37^{\circ} \mathrm{C}$. Also, PCR result displays a single bright specific band on the agarose gel using the genomic DNA as the template to amplify $16 \mathrm{~S}-23 \mathrm{~S}$ sequence after stored at $4^{\circ} \mathrm{C}$ and $37^{\circ} \mathrm{C}$, respectively (Figure 7), indicating that the property of genomic DNA reference materials is good.

\subsection{Long-Term Stability Analysis of Genomic DNA Reference} Material of C. sakazakii. After the genomic DNA reference materials were lyophilized, they were stored at $-20^{\circ} \mathrm{C}$. For each test of the long-term stability test, 3 bottles of samples were randomly taken out of the lyophilized samples. At the 1st, 2nd, 4th, 6th, and 8th month, a total of 5 time points were sampled and tested. The content changes among the samples at different time points are shown in Figure 8. The $F$ value of 0.696 was less than the critical value of 1.66 at $95 \%$ confidence, showing that the genomic DNA reference material was stable for 8 months stored at $4^{\circ} \mathrm{C}$ or $-20^{\circ} \mathrm{C}$. Figure 9 shows the results of PCR of the characteristic sequence $16 \mathrm{~S}-23 \mathrm{~S}$ of each sample. The characteristic gene test is positive, and the bands of PCR products were single, indicating that the sample is stable for 8 months.
3.7. Detection of Foodborne Pathogen C. sakazakii in Flammulina velutipes. Fifteen batches of flesh Flammulina velutipes were purchased from supermarkets and traditional retail markets spread over Jinan. The samples were detected immediately while they arrived in the laboratory. The results showed that 16S-23S fragments of C. sakazakii were positive in all tested samples from 8 supermarkets (Figure 10) and 5 positive of 7 samples from traditional retail markets (Figure 11$)$. In total, the detection rate was $86.7 \%$.

3.8. Verification of the PCR Detection of C. sakazakii in Flammulina velutipes. The positive samples were verified according to the National Food Safety Standard of China Food microbiological examination: Enterobacter sakazakii (GB 4789.40-2010; National Standard of the People's Republic of China, 2010). As shown in Figure 12, the colonies of no. 1 bacterium isolated from Flammulina velutipes on TSA plate were yellow and on the chromogenic Enterobacter sakazakii agar plate were blue-green (other samples were not shown). The biochemical identification results are shown in Table 3, which were consistent with the positive control of C. sakazakii FC4146. All the results indicated that the bacterium detected in Flammulina velutipes is C. sakazakii.

\section{Discussion}

In this study, genomic DNA reference materials were prepared by genomic DNA extraction and lyophilization. The uniformity analysis showed that the samples have no significant differences among samples, and they were stable at $4^{\circ} \mathrm{C}$ or $37^{\circ} \mathrm{C}$ for 14 days, which could meet the requirements of long-distance transportation. The samples keep good as long as 8 months while stored at $4^{\circ} \mathrm{C}$ or $-20^{\circ} \mathrm{C}$. The reference materials were used as the positive control in the detection of Cronobacter sakazakii of edible fungus Flammulina velutipes. This reference material is suitable for the verification, confirmation, and evaluation of PCR detection methods of Cronobacter spp. in food and other samples, quality control of the detection process, personnel assessment, evaluation of laboratory detection capabilities, and interlaboratory comparisons. Also, this reference material could be used as a quantitative tool for Cronobacter spp. using other detective methods. For example, a standard curve could be formed based on this reference material using fluorescence quantitative PCR analysis, and the pathogenic bacteria content of the sample to be tested 
$4^{\circ} \mathrm{C}$

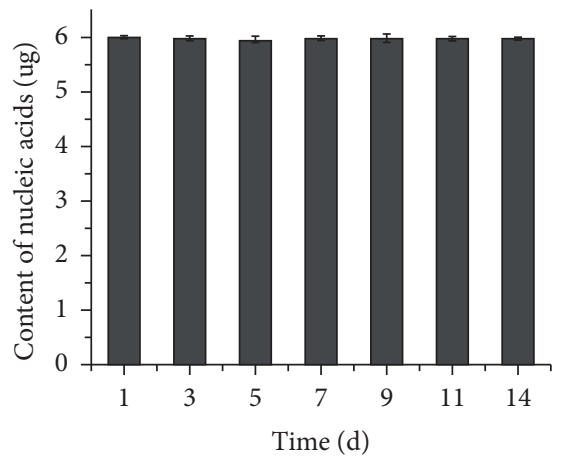

(a) $37^{\circ} \mathrm{C}$

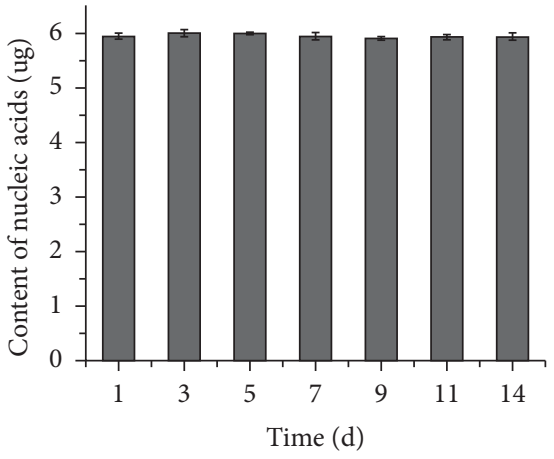

(b)

FIGURE 6: The short-term stability test of changes in 14 days of genomic nucleic acid reference material of C. sakazakii.

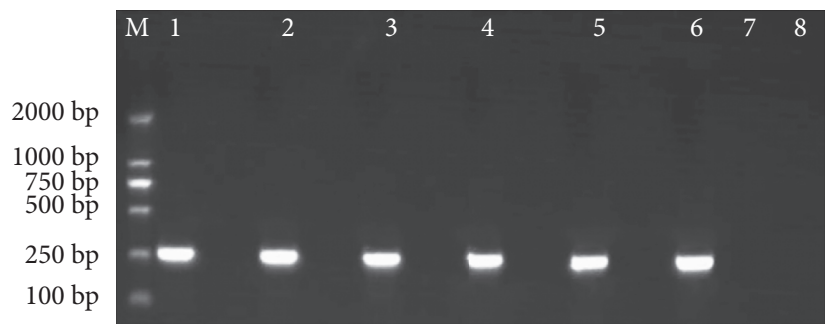

FIGURE 7: The PCR analysis of the short-term test of genomic DNA reference materials for 14 days. $M$, DNA marker D (100 2000 bp); lanes $1-3$, PCR results of 3 samples stored at $4^{\circ} \mathrm{C}$, respectively; lanes $4-6$, PCR results of 3 samples stored at $37^{\circ} \mathrm{C}$, respectively; lane 7 , negative control; and lane 8 , blank control.

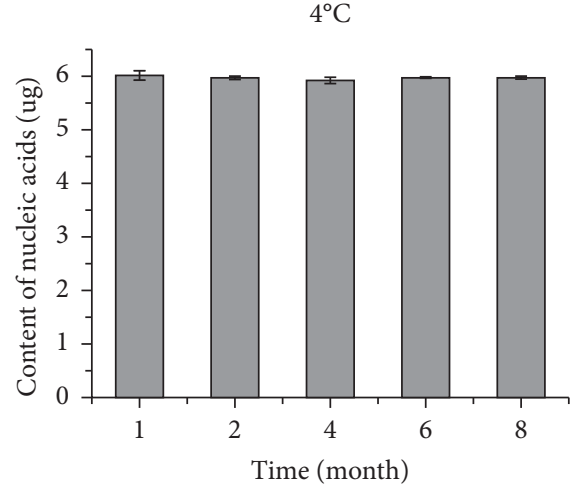

(a)

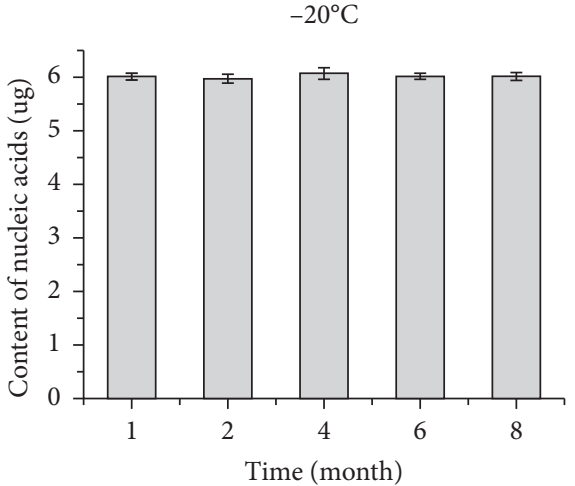

(b)

Figure 8: The long-term stability test of changes in 8 months of genomic nucleic acid reference material of C. sakazakii.

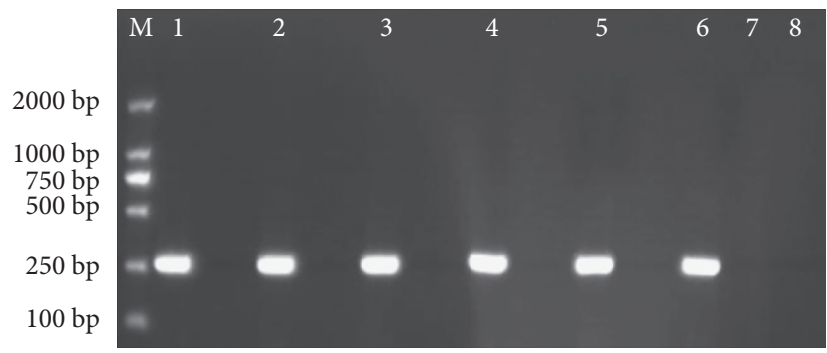

Figure 9: The PCR analysis of the long-term test of genomic DNA reference materials for 8 months. $M$, DNA marker D (100 2000 bp); lanes 1-3, PCR results of 3 samples stored at $-20^{\circ} \mathrm{C}$, respectively; lanes $4-6$, PCR results of 3 samples stored at $4^{\circ} \mathrm{C}$, respectively; lane 7 , negative control; and lane 8 , blank control. 


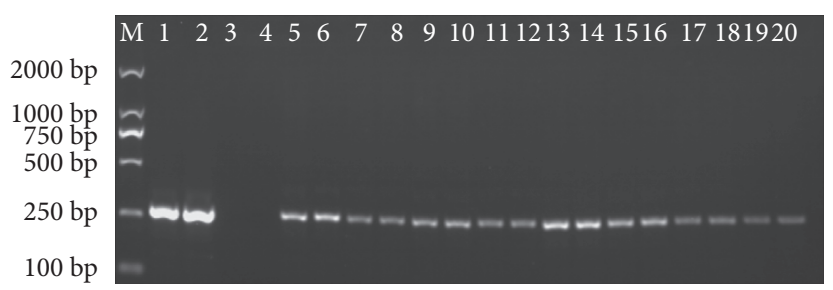

Figure 10: PCR detection of 16S-23S of C. sakazakii in Flammulina velutipes collected from 8 supermarkets. M, DNA marker D (100 2000 bp); lanes 1-2, positive control; lane 3, negative control; lane 4, blank control; and lanes 5-20, PCR results of 8 samples, respectively; each sample was tested twice.

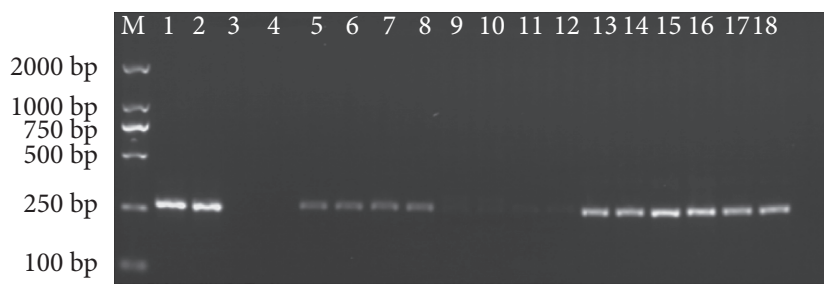

FIGURE 11: PCR detection of 16S-23S of C. sakazakii in Flammulina velutipes collected from 7 traditional retail markets. $M$, DNA marker D (100 2000 bp); lanes 1-2, positive control; lane 3, negative control; lane 4, blank control; and lanes 5-18, PCR results of 7 samples, respectively; each sample was tested twice.

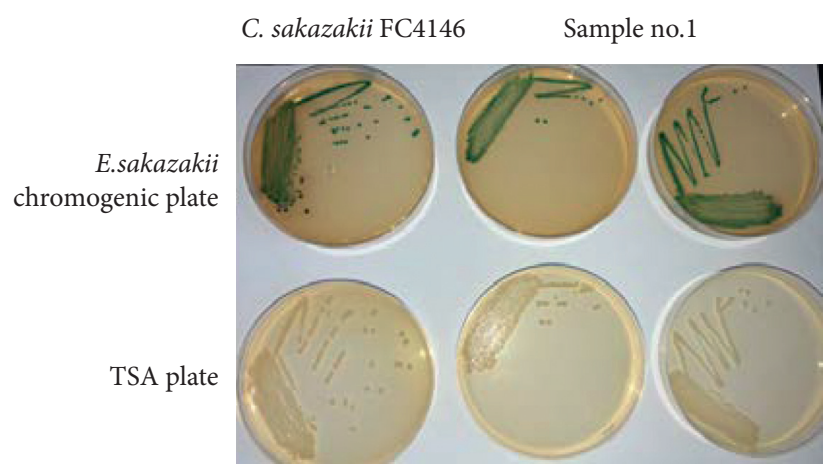

Figure 12: The colonies of C. sakazakii and the no. 1 bacterium detected in Flammulina velutipes.

TABle 3: The biochemical identification of the bacterium detected in Flammulina velutipes.

\begin{tabular}{|c|c|c|c|c|c|c|c|c|c|c|c|c|c|c|}
\hline Sample no. & 1 & 2 & 3 & 4 & 5 & 6 & 7 & 8 & 9 & 10 & 11 & 12 & 13 & C. sakazakii FC4146 \\
\hline Oxidase & - & - & - & - & - & - & - & - & - & - & - & - & - & - \\
\hline L-lysine decarboxylase & - & - & - & - & - & - & - & - & - & - & - & - & - & - \\
\hline L-ornithine decarboxylase & + & + & + & + & + & + & + & + & + & + & + & + & + & + \\
\hline L-arginine dihydrolase & + & + & + & + & + & + & + & + & + & + & + & + & + & + \\
\hline Citric acid hydrolysis & + & + & + & + & + & + & + & + & + & + & + & + & + & + \\
\hline Sorbitol & - & - & - & - & - & - & - & - & - & - & - & - & - & - \\
\hline D-rhamnose & + & + & + & + & + & + & + & + & + & + & + & + & + & + \\
\hline D-sucrose & + & + & + & + & + & + & + & + & + & + & + & + & + & + \\
\hline D-melibiose & + & + & + & + & + & + & + & + & + & + & + & + & + & + \\
\hline Amygdalin & + & + & + & + & + & + & + & + & + & + & + & + & + & + \\
\hline
\end{tabular}

can be judged according to the standard curve applied in the same analysis method. No matter what the PCR method is, there are limitations; sometimes, false-positives are very common using PCR methods. In actual food detection applications, PCR detection methods can effectively and quickly exclude a large number of negative result samples, saving time and cost. A positive PCR result is only a preliminary result. In the end, culture and biochemical identifications are required to determine whether the sample contains the target pathogen, as we show in our study above. The development of this research is conducive to the popularization and application of genomic nucleic acid reference materials in rapid foodborne microbial detection. 


\section{Data Availability}

The data used to support the findings of this study are available from the corresponding authors upon request.

\section{Conflicts of Interest}

The authors declare that they have no conflicts of interest.

\section{Authors' Contributions}

Huandie Yang and Danyang Guo are co-first authors. Huandie Yang and Danyang Guo conducted the experiments; Leilei Chen and Alexander Suvorov offered the program funding; Qingxin Zhou, Junhua Wang, and Xiaofeng Gao revised the manuscript; Jinyu Yang and Leilei Chen directed the work; Jinyu Yang wrote the manuscript; and Xiangyan Chen supervised the work.

\section{Acknowledgments}

This research was supported by the National Key Research and Development Program of China (2017YFC1601400), Shandong Provincial Key Research and Development Program (2019GHZ022), Taishan Scholar Program of Shandong Province (tsqn201909168), and "Double Hundred" Program for Foreign Experts of Shandong Province (WST2017004).

\section{Supplementary Materials}

Table S1. PCR system for detection of characteristic 16S-23S sequence of C. sakazakii. Table S2. PCR reaction program for detection of characteristic 16S-23S sequence of C. sakazakii. (Supplementary Materials)

\section{References}

[1] S. Umesha and H. M. Manukumar, "Advanced molecular diagnostic techniques for detection of food-borne pathogens: current applications and future challenges," Critical Reviews in Food Science and Nutrition, vol. 58, no. 1, pp. 84-104, 2018.

[2] J. Bai, Y.-T. Kim, S. Ryu, and J.-H. Lee, "Biocontrol and rapid detection of food-borne pathogens using bacteriophages and endolysins," Frontiers in Microbiology, vol. 7, p. 474, 2016.

[3] B. P. Blackwood and C. J. Hunter, "Cronobacter spp," Emerging Infections 10, vol. 4, no. 2, p. 255, 2016.

[4] M. Liu, G. Hu, Y. Shi et al., "Contamination of Cronobacter spp. in chinese retail spices," Foodborne Pathogens and Disease, vol. 15, no. 10, pp. 637-644, 2018.

[5] S. J. Forsythe, "Updates on the cronobacter genus," Annual Review of Food Science and Technology, vol. 9, no. 1, pp. 23-44, 2018.

[6] M. Friedemann, "Epidemiology of invasive neonatal Cronobacter (Enterobacter sakazakii) infections," European Journal of Clinical Microbiology \& Infectious Diseases, vol. 28, no. 11, pp. 1297-1304, 2009.

[7] L. K. Dygico, C. G. M. Gahan, H. Grogan, and C. M. Burgess, "The ability of listeria monocytogenes to form biofilm on surfaces relevant to the mushroom production environment," International Journal of Food Microbiology, vol. 317, Article ID $108385,2020$.
[8] M. Chen, Q. Wu, J. Zhang, W. Guo, S. Wu, and X. Yang, "Prevalence and contamination patterns of Listeria monocytogenes in Flammulina velutipes plants," Foodborne Pathogens and Disease, vol. 11, no. 8, pp. 620-627, 2014.

[9] Y. Ye, H. Li, Q. Wu, M. Chen, Y. Lu, and C. Yan, "Isolation and phenotypic characterization of Cronobacter from dried edible macrofungi samples," Journal of Food Science, vol. 79, no. 7, pp. M1382-M1386, 2014.

[10] C. Li, H. Zeng, J. Zhang et al., "Prevalence, antibiotic susceptibility, and molecular characterization of Cronobacter spp. isolated from edible mushrooms in China," Frontiers in Microbiology, vol. 10, p. 283, 2019.

[11] A. Souii, M. B. M'hadheb-Gharbi, and J. Gharbi, "Nucleic acid-based biotechnologies for food-borne pathogen detection using routine time-intensive culture-based methods and fast molecular diagnostics," Food Science and Biotechnology, vol. 25 , no. 1 , pp. 11-20, 2016.

[12] T. Notomi, H. Okayama, H. Masubuchi et al., "Loop-mediated isothermal amplification of DNA," Nucleic Acids Research, vol. 28, no. 12, pp. 63e-63, 2000.

[13] S. Chattopadhyay, A. Kaur, S. Jain, and H. Singh, "Sensitive detection of food-borne pathogen Salmonella by modified PAN fibers-immunoassay," Biosensors and Bioelectronics, vol. 45, pp. 274-280, 2013.

[14] Y. Liu, Y. Cao, T. Wang, Q. Dong, J. Li, and C. Niu, "Detection of 12 common food-borne bacterial pathogens by TaqMan real-time PCR using a single set of reaction conditions," Frontiers in Microbiology, vol. 10, p. 222, 2019.

[15] National Standard of China, National Food Safety Standard of China, Food Microbiological Examination: Enterobacter sakazakii for Powdered Infant Formula, Vol. GB 4789.40-2010, National Standard of China, Beijing, China, 2010. 